\title{
Upgrade of the YerPhl polarization Lidar System for using Polarization of the Elastic and Raman Backscattered Beams.
}

\author{
A. Ghalumyan ${ }^{1, *}, K$. Apresyan ${ }^{1}, A$. Chilingaryan $^{1}$, and $V$. Ghazaryan ${ }^{1}$ \\ ${ }^{1}$ A. Alikhanyan National Lab (Yerevan Physics Institute), Yerevan, Armenia.
}

\begin{abstract}
A powerful two-frequency lidar system using polarized beams has been developed at YerPhI. The system is completed with laser beam polarization changers and nitrogen and water Raman channels for investigation of the influence of atmospheric electric fields on the elastic and Raman backscattered beams polarization. At present, the system is being tuned for measuring vertical atmospheric backscatter profiles of aerosols and hydrometeors, analyze the depolarization ratio of elastic backscattered laser beams and investigate the influence of external factors on the beam polarization. Laser light that is reflected from the air and from clouds carries information on density profiles, aerosols and electrical fields. Applications of this system will be the investigation of the electrical state of the atmosphere during thunderstorms [1-3] on Mt Aragats, and, possibly the monitoring of the atmosphere at the site of the upcoming Cherenkov Telescope Array (CTA). CTA is expected to provide unprecedented sensitivity for gamma ray detection in the energy range of $30 \mathrm{GeV}$ to $300 \mathrm{TeV}$. To fully exploit the potential of the telescope system it is important to characterize the optical and electrical properties of the atmosphere. A lidar system for the continuous monitoring of the atmosphere is the tool of choice.
\end{abstract}

\section{The LIDAR technique basics}

Earth atmosphere, including suspending aerosols inside, have a variety of impacts which directly affect the radiative balance of the Earth-atmosphere system through scattering and absorption. The aerosols, being the most variable component in the atmosphere, need to be constantly monitored as they reflect the solar radiation at a daytime to decrease the overheating of the Earth surface and retain the infrared near the ground in the night time.

The polarization LIDAR technique has the unique ability to discriminate between spherical and irregular particles and identify the type of atmospheric aerosols because of its sensitivity to particle shape [4]. The polarization state of the backscattered beam can be used to differentiate solid and liquid particles (ice and water, etc).

One of the most useful experimental techniques involves the use of a linearly polarized lidar output and a receiver capable of simultaneously measuring the backscattered light two polarization components oriented parallel $\left(P_{\|}\right)$and perpendicular $\left(P_{\perp}\right)$ to the direction of the laser beam polarization axis. The linear depolarization ratio $\delta=P_{\perp} / P_{\|}$can be measured as an indicator of the polarization changes.

The lidar equation in the simplest form can be written as:

$$
P(R)=\frac{K P_{L}}{R^{2}} \beta(R) \exp (-2 \alpha R)
$$

where, $P(R)$ is the power received from a distance $\mathrm{R}, K$ summarizes the lidar efficiency, $P_{L}$ is the laser power, $\beta(R)$

\footnotetext{
*e-mail: ghalumyan@yandex.ru
}

is the backscatter coefficient and $\exp (-2 \alpha R)$ is the atmospheric attenuation.

The measured data by means of the LIDAR System can be written as:

$$
\begin{gathered}
\frac{P(R)}{K P_{L}} R^{2}=\beta(R) \exp (-2 \alpha R) \\
\delta(R)=\frac{P_{\perp}(R)}{P_{\|}(R)}
\end{gathered}
$$

\section{The developed LIDAR System}

The LIDAR system consists of an optical unit, including the Laser Emitter (LE), receiving system and PMTs, and an electronic unit, including: LE control, data processing and control systems (Figure 1 and Figure 2).

The LE of the polarized laser radiation is a custom made solid state, flash-lamp pumped, Q-switched YAG: $\mathrm{Nd}^{3+}$ laser with a second harmonic generator (SHG) and a Beam Expander (BE) [5, 6]. It generates linearly polarized $10 \mathrm{~ns}$ pulses with $1064 \mathrm{~nm}$ and/or $532 \mathrm{~nm}$ wavelengths and a repetition rate of $10-20 \mathrm{~Hz}$.

The LE is equipped with motorized positioning system of polarization optics, by means of which its output beam polarization can be remotely changed for both wavelengths of $1064 \mathrm{~nm}$ and $532 \mathrm{~nm}$, including: both parallel linear horizontal or vertical; both parallel linear 45 degrees; linear and circular etc.

The laser oscillator and amplifier flash-lamps are connected in series and are operated by means of one custom made flash-lamp driver (FLD). The FLD includes a simmer supply and capacitor charging power supply and generates $120 \mu$ s pulses of 20-60 J energy into flash-lamps 


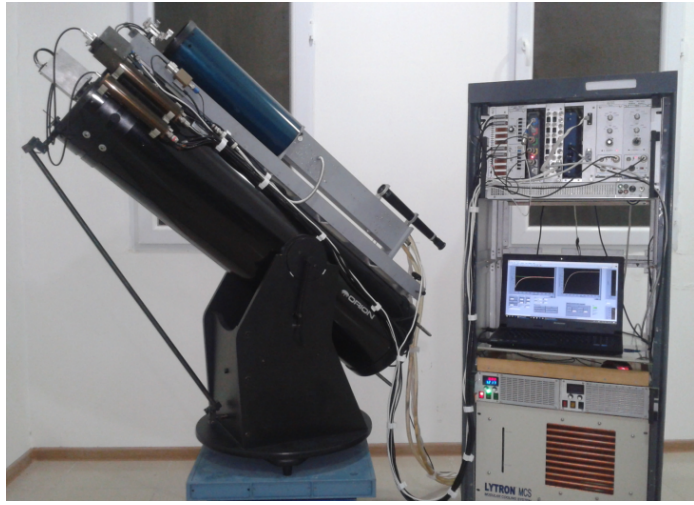

Figure 1. The LIDAR System.

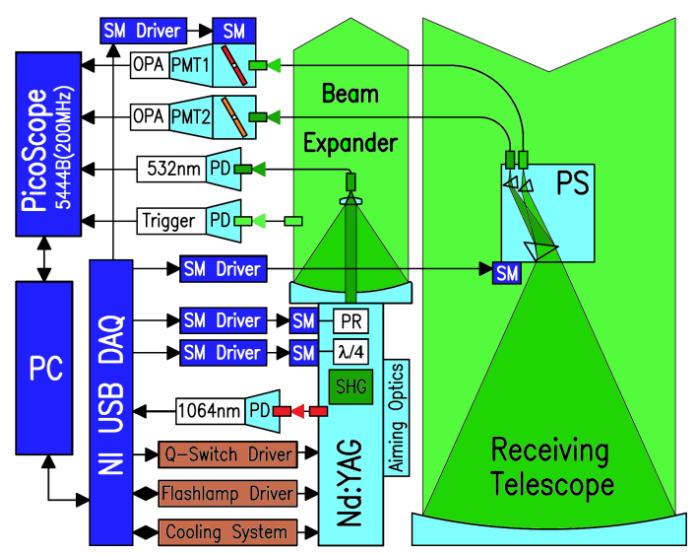

Figure 2. The LIDAR System schematic diagram.

with repetition rate of $10-20 \mathrm{~Hz}$. It has such protection features as overcharge, overheat, flash-lamp breakdown, coolant overheat, coolant flow interruption, etc.

The BE is an extra cavity Cassegrain telescope with $12^{X}$ magnification, which allows to expand the $8 \mathrm{~mm}$ across laser output beam to about $100 \mathrm{~mm}$ diameter and reduce the laser beam divergence down to $<10^{-4} \mathrm{rad}$.

Receiving System of backscattered radiation contains 250 $\mathrm{mm}$ aperture Receiving Telescope (RT) and a custom made Polarization Separator (PS). The PS is placed on the output of the RT and its function is to separate the orthogonal to each other polarization components of the backscattered signals. To reduce an additional depolarization of the signals in the receiving optics, the PS was designed without optical components before polarization separation. For polarization separation is used a Calcite prism and two prisms with different refractive indexes reducing aberrations of the separated signals on the focus of RT mirror. For this configuration the signals with orthogonal to each other polarization have been refracted under different angles and separated from each other in space for more than $8 \mathrm{~mm}$.

The parallax between the laser beam and the receiver is $270 \mathrm{~mm}$. It is expected from ray tracing simulations to be able to have a complete overlap of the LE beam and the mirror field of view at a distance of about $130 \mathrm{~m}$. By
Table 1. The LIDAR System Specifications.

\begin{tabular}{|l|l|}
\hline Laser Emitter & \\
\hline Laser Source & YAG:Nd ${ }^{3+}$ Custom made \\
\hline Wavelength & $1064 \mathrm{~nm}$ and/or 532 nm \\
\hline Pulse energy & $300-700 \mathrm{~mJ} @ 1064 \mathrm{~nm}$ \\
& $100-250 \mathrm{~mJ} @ 532 \mathrm{~nm}$ \\
\hline Beam Expander & $12^{x}$ \\
\hline Beam divergence & $<0.1 \mathrm{mrad}$ (after BE) \\
\hline Pulse width & $10 \mathrm{~ns}$ \\
\hline Repetition rate & $10-20 \mathrm{~Hz}$ \\
\hline Polarization linearity & $<10^{-3}$ \\
\hline Receiving Telescope & \\
\hline Diameter & $250 \mathrm{~mm}$ \\
\hline Field of view & $1.6 \mathrm{mrad}$ \\
\hline Polarization Separator & Custom made \\
\hline Fiber bundle aperture & $3 \mathrm{~mm}$ \\
\hline PMT & FEU-100 and FEU-83 \\
\hline Range & $0.15-15 \mathrm{~km}$ \\
\hline Data processing & \\
\hline Oscilloscope & PicoScope 5444B \\
\hline DAQ & NI USB DAQ \\
\hline Software interface & LabVIEW \\
\hline
\end{tabular}

means of the RT mirror the backscattered radiation is directed and focused on the inputs of the $3 \mathrm{~mm}$ aperture fiber bundles. On its way the radiation is passing through PS which separates orthogonal to each other polarized components of the backscattered radiation. Two fiber bundles transport the separated optical signals to PMT boxes. Signals, after optical filtering from background noise by means of narrow band filters, enter into PMTs, which amplifies and converts them into electrical signals.

The electrical signals from PMTs passed to the signal processing system. The signals digitizing are realized by means of $200 \mathrm{MHz}$ PicoScope 5444B. The triggering of the registration system was organized by means of photodiode system optically communicated with outgoing laser pulse. The Laser output beam power for both wavelengths also was measured by means of photodiodes and digitized. The angle of the PS can be controlled by means of stepper motor (SM) and play-free gear allowing orient the polarization separation angle perpendicular to LE beam polarization plane as well, as to any angle to it. The signal processing and storage, as well as the control of all LIDAR subsystems, is done by means of a PC. The CAMAC crate is used as a chassis for custom made blocs and as a power supply for different LIDAR subsystems and modules. The custom made power supplies for system electronics, NI USB DAQ system, custom made BNC module for DAQ inputs and outputs, PicoScope 5444B, SM driver, PMT power supplies, etc. are installed into CAMAC crate (Figure 1). To connect and control all the subsystems and subprograms with one graphical user interface, the LabVIEW is used.

The all LIDAR control subsystems, including LE FLD and cooling system are installed into one rack. They are connected with optical unit with two separated in space cable bundles reducing the influence of LE flash-lamp discharge 


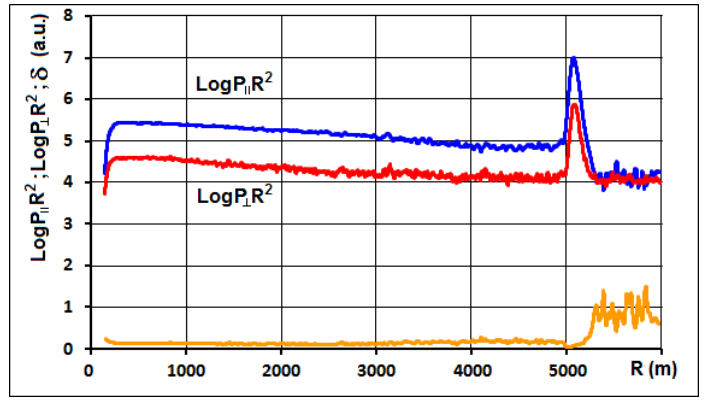

Figure 3. Range normalized polarization separated backscatter profiles and depolarization ratio profile.

electromagnetic noise on the registered signals.

The specifications of the LIDAR System are indicated in Table 1.

\section{The Measurements of the Backscattered Profiles}

The backscattered profiles, presented on Figure 3 are measured at summer nighttime at zenith angle of 60 degrees with digitizing resolution of about $1 \mathrm{~m}$. The signals are normalized on LE beam outputs and averaged by 10 .

On the figures one can notice that the depolarization of the backscattered laser radiation in the clouds is small, which is an indication of the cloud consistency mainly of spherical aerosols. From the profiles can be easily calculated the heights, the thickness and distribution of the cloud by measuring the attenuation of the laser radiation in the clouds, and so on.

These measurements of the backscattered signal profiles from the atmosphere and clouds by means of the designed LIDAR system shows that it can be used in the numerous applications of atmospheric research.

Now the LIDAR is in process of calibration for investigation of the static electric fields in the atmosphere and clouds. The measurements will be carried out by linear depolarization ratio measurements of the polarized backscattered laser beam.

The polarization of the laser beam, passing through atmosphere, can be changed by means of two factors. The first - 1t can be depolarized by means of backscattering from irregular particles of aerosols etc. The second - it can be depolarized passing through the static electric field due to Kerr effect (Figure 4).

where, $E$ is the electric field, $P_{L}$ is the laser polarization, $P_{\|}, P_{\perp}$ and $P_{E}$ are the parallel to laser beam, the depolarized by the atmosphere and the rotated by electric field polarization components of the backscattered radiation correspondingly.

The influence of the electric field on the depolarization of the laser beam exists when the electric field direction have an angle with the laser beam polarization plane and is maximal if the angle is 45 degrees. For measurement of the electric field strength the first factor is playing a role of noise in backscattered signals. So the measurement of the electric field strength and direction can be performed by in
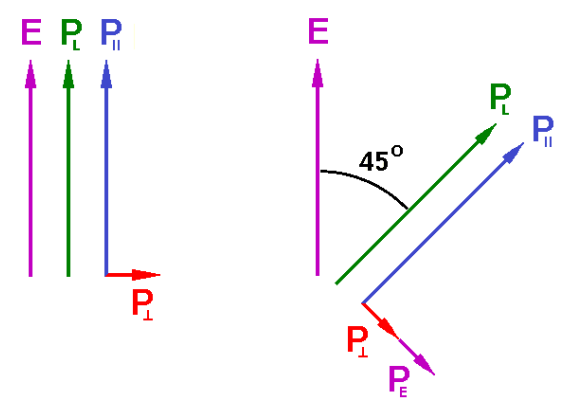

Figure 4. Kerr effect for electric field observations.

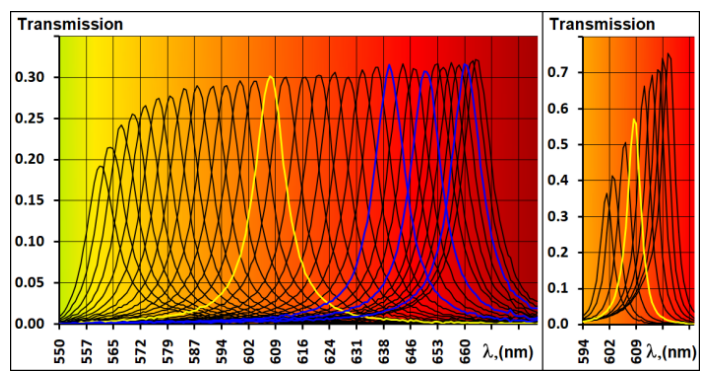

Figure 5. Stepper driven tunable and manually adjustable narrow band filters transmission (3step/curve).

series measurements of the values of backscattered beam linear depolarization ratios for different LE beam polarization planes (particularly for vertical and 45 degrees to it planes). The difference of these two measurements will contain information about electric field strength and direction.

\section{Water and Nitrogen Raman channels}

LIDAR system PMTs can be equipped with narrow band filters for polarization measurements on the laser $532 \mathrm{~nm}$ wavelength as well, as with tunable narrow band filters allowing to investigate depolarization ratio profiles on the different wavelengths including nitrogen Raman shifts and Raman shifts of the water in three phases (ice, liquid, and vapor) and investigate the influence of external factors (including static electric fields) on the shape of the $3400 \mathrm{~cm}^{-1}$ water Raman band.

One of the narrow band filters with spectral bandwidth of $10 \mathrm{~nm}$ (FWHM) can be remotely tuned by means of SM in $560 \mathrm{~nm}-664 \mathrm{~nm}$ wavelengths region with scanning accuracy of $2 \mathrm{~nm}-0.73 \mathrm{~nm}$. It can be used for registration of Raman spectrum of water in clouds as well as for distinguishing of water three different phases.

The second filter with spectral bandwidth of $4.5 \mathrm{~nm}$ (FWHM) is manually adjustable in $601 \mathrm{~nm}-618 \mathrm{~nm}$ region with accuracy of $0.5 \mathrm{~nm}$. It can be used as nitrogen channel for registration of $2330 \mathrm{~cm}^{-1}$ Raman shifted bands $(\mathrm{O}, \mathrm{Q}, \mathrm{S})$ of atmospheric nitrogen. The transmission characteristics of both filters are indicated on Figure 5. The figure is simplified by removing intermediate $2 / 3$ part of the curves ( 3 steps/curve). Besides whole scanning wave- 
length range filters transmission spectral bandwidths are constant. Yellow curves indicate the nitrogen $2330 \mathrm{~cm}^{-1}$ Raman shifts and the blue ones indicate the centers of the ice, liquid water and water vapor Raman bands.

Due to the fact that narrow band filters transmission are angular dependent, after signal transportation by fiber bundles, before passing the filters, the optical beams are collimated by means of optical lens systems. The maximum beam spot diameter along the optical path in the filter boxes up to the PMTs are approximately $14 \mathrm{~mm}$, and the beam divergence is less than 4 mrad.

\section{Summary}

A polarization LIDAR system for remote sensing of the atmosphere was designed at the Yerevan Physics Institute and is used for polarization separated backscatter profiles measurements in the atmosphere. It has capabilities of changing the Laser output beam polarization as well as to analyze the polarization state of received signals for any angle.

Now it is directed to investigate the influence of the exter- nal factors on the backscatter signals polarization, including influence of the atmospheric electric fields.

The system is completed with nitrogen and water Raman channels into the receiver to investigate the cloud and aerosol backscatter and extinction, as well, as the influence of the external factors (including static electric fields) on the deformation of the spectral band of water.

\section{References}

[1] Chilingarian A., Daryan A., Arakelyan K. et. Al., Phys. Rev. D, 82, 043009 (2010).

[2] Chilingarian A., Hovsepyan, G., Hovhannisyan, A., Phys. Rev. D: Part. Fields, 83 (6), 062001 (2011).

[3] Chilingarian A., B. Mailyan B., Vanyan L., Atmos. Res., 1, 114-115 (2012).

[4] Sassen K., Bull. Am. Meteorol. Soc., 12, 1848 (1991).

[5] Ghalumyan A.S., Ghazaryan V.R., Proceedings of the International Symposium TEPA-2015, ed. Chilingarian A., p. 28 (2015).

[6] Apresyan K.G., Ghalumyan A.S., Ghazaryan V.R. . International Conference LP-2017, Armenia. 